\title{
Regulation of a Periodic Motor Program in C. elegans
}

\author{
Dennis WC. Liu and James H. Thomas \\ University of Washington, Department of Genetics, Seattle, Washington 98195
}

\begin{abstract}
A three-part motor program mediates a defecation every $\mathbf{4 5}$ sec in well-fed wild-type Caenorhabditis elegans. Individual worms maintain this $\mathbf{4 5} \mathrm{sec}$ rhythm with an SD of about 3 sec. We present evidence that the defecation cycle is controlled by an endogenous clock, most likely a neuronal pattern generator. The phase of the behavioral rhythm can be reset like pattern generators in other animals. The rhythm was reset by stimulating a well-characterized neuronal circuit mediating response to light touch. Also, animals that spontaneously stopped feeding interrupted their defecation rhythms. When they resumed feeding these animals reactivated the motor program in phase with the previously established rhythm, indicating that an endogenous clock continues to run even when the behavior is not expressed.

Control of the defecation rhythm is independent of expression of the motor program. Most previously isolated mutations that affect the motor program (Thomas, 1990) do not alter the rhythm of the behavior; the motor steps themselves are defective but not the timing of their activation. Laser kills of identified motor neurons that affect particular parts of the motor program also did not change the defecation rhythm.

Another sensory stimulus, food, strongly modulates defecation behavior: animals away from food rarely activated the motor program, and food dilution resulted in a graded lengthening of the cycle period. To elucidate further the relationship between feeding and defecation rhythms we studied a mutation, dec-8(sa200), that caused worms to continue to activate the motor program in the absence of food. The mutant did not require the presence of food to activate the motor program, although food made the rhythm more precise. In the presence of food, dec-8(sa200) animals exhibited tandem activations of the defecation motor program; the principal activation was followed by a more variable second activation. Further experiments suggested that the tandem activations of the motor program are not due to the activity of multiple oscillators.
\end{abstract}

[Key words: biological rhythms, pattern generator, fixed action pattern, behavioral genetics, motor control, biological clock, neuroethology, phase response, motor program, cellular oscillator]

\footnotetext{
Received July 12, 1993; accepted Sept. 14, 1993.

We thank Jon Yeagers for indispensable help in developing the computer programs for recording and displaying behavioral data. Special thanks to David J. Reiner for generously sharing some laser-operated animals and for comments on the manuscript. For critical comments on the manuscript, thanks to Dr. John Edwards and Dr. Judith Eisen.

Correspondence should be addressed to Dennis WC. Liu, Ph.D., Department of Genetics, SK50, University of Washington, Seattle, WA 98195.

Copyright (c) 1994 Society for Neuroscience $0270-6474 / 94 / 141953-10 \$ 05.00 / 0$
}

Behavioral patterns generally have two types of temporal organization. First, groups of muscles are coordinately activated to produce a coherent motor program (von Holst, 1937; Gray, 1939). Second, as we emphasize in this report, the timing of a motor program's activation is important as well, so that muscles contract at the appropriate moment and the right number of times. Internal and external factors can play a role in determining when a behavioral pattern is triggered (Lorenz and Tinbergen, 1938). Most biological rhythms, although affected by the external environment, arise principally from endogenous conditions. Examples include cell-division cycles (Edmonds and Adams, 1981), heartbeats (Hoyle, 1982), peristaltic intestinal contractions (Maeda et al., 1992), slime-mold cytoplasmic streaming (Smith and Saldana, 1992), estrous cycles (Austin and Short, 1972), and circadian rhythms (Binkley, 1990). In the case of cell-division cycles and circadian rhythms, molecular genetic approaches have yielded insights into the molecular mechanisms underlying the biological rhythms. We have been studying a periodically activated motor program in Caenorhabditis elegans to develop a system for molecular genetic analysis of shortperiod cellular oscillators.

In the nematode $C$. elegans, defecation is achieved by the regular activation of a highly stereotyped motor program controlling the sequential activation of three sets of muscles (Croll and Smith, 1978; Thomas, 1990). Our goal has been to understand the environmental and internal factors that control the periodic activation of the motor program. The defecation motor program (DMP) begins with the simultaneous contraction of posterior body-wall muscles near the tail, pressurizing gut contents and pushing them forward. The posterior body-wall muscles then relax and about $2 \mathrm{sec}$ later anterior body-wall muscles simultaneously contract, forcing the pharynx back against the intestinal lumen. Immediately following (sometimes coincident with) the anterior contraction, muscles that open the anus contract to release the pressurized gut contents (Thomas, 1990). This entire sequence of muscle contractions takes about $5 \mathrm{sec}$. In wild-type animals the DMP is activated just once for each defecation cycle. The activation is frequent and highly regular, occurring every $45 \mathrm{sec}$, typically with an SD of about $3 \mathrm{sec}$ for a well-fed adult worm (Croll and Smith, 1978; Thomas, 1990).

To understand the nature of the pattern generator controlling activation of the DMP, we undertook a detailed behavioral analysis of the wild-type worms and of several behavioral mutants of $C$. elegans. Various factors might determine when the motor program is activated, and we weigh two alternative explanations: first, whether the rhythm of DMP activation is generated by a reflex response to a stimulus such as food sensation or gut distention; and second, the alternative explanation that DMP rhythms are the product of an endogenous pattern generator. Our results demonstrate that while food and other sen- 

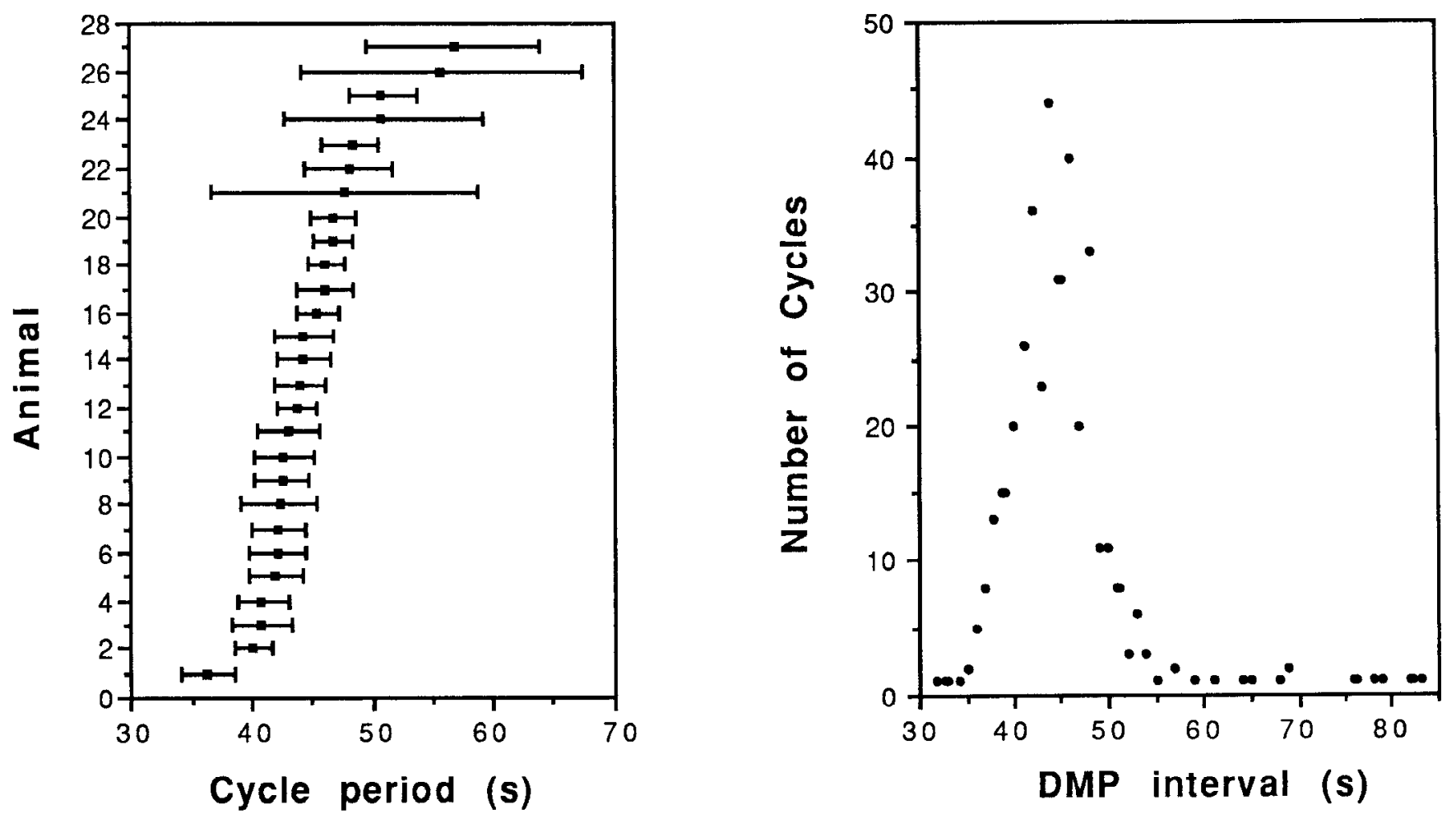

Figure 1. Activation of the defecation motor program is highly periodic. Left, The distribution of average defecation periods for 27 individual animals assayed for at least 10 min each. The average periods ranged from 36.3 to $56.8 \mathrm{sec}$. The mean for the average periods for all 27 animals was 45.3 , with an SD of $4.3 \mathrm{sec}$. Error bars represent $1 \mathrm{SD}$ to each side of the average. Right, The distribution of defecation intervals for 377 cycles in the same 27 animals. The distribution strongly clustered around the mean of $45.1 \mathrm{sec}$. The shortest interval was 31.6 sec and the longest was $83.3 \mathrm{sec}$.

sory stimuli powerfully modulate defecation periodicity, an endogenous clock ultimately generates the behavioral rhythm.

\section{Materials and Methods}

Animals. All animals used in these experiments were of the species Caenorhabditis elegans and were derived from the N2 Bristol wild-type laboratory strain (Brenner, 1974). The strains used were N2, dec-8(sa200), and aex-2(sa2l). Stocks were raised on monoxenic OP50 bacteria, a leaky uracil-requiring strain of $E$. coli. Most experiments were on standard $5 \mathrm{~cm} \mathrm{NG}$ agar plates with thick bacterial lawns (Brenner, 1974). Stocks of worms were on these plates at $20^{\circ} \mathrm{C}$ unless otherwise stipulated.

Identification of the dec-8(sa200) mutation. The phenotype of dec$8(\mathrm{sa200)}$ animals was first noticed in a strain carrying daf $19(\mathrm{~m} 86)$ (Thomas, 1990). Subsequent outcrossing and mapping showed that the defecation phenotype (tandem activations of the motor program) did not cosegregate with daf-19 mutant phenotypes, dec-8(sa200) mapped instead to the cluster of chromosome IV (D. WC. Liu and J. H. Thomas, unpublished observations). All the reported observations of $d e c-8 \mathrm{mu}-$ tant animals were done on an outcrossed strain (JT200) wild type for the daf- 19 locus.

Recording defecation cycles. Prior to making behavioral observations plates of worms were moved from their cultured temperature of $20^{\circ} \mathrm{C}$ to room temperature $\left(22-24^{\circ} \mathrm{C}\right)$ for at least $60 \mathrm{~min}$, unless otherwise noted. Defecation behavior was observed on worms that had been moved alone or in a small group to a fresh plate and allowed to settle for at lcast $5 \mathrm{~min}$ prior to collecting data. The uncrowded plate made it easier to follow a single worm over time and minimized interactions with other worms. The three muscle contractions that comprise the defecation motor program, posterior body-wall muscle contraction (p), anterior body-wall muscle contraction (a), and expulsion muscles contraction $(x)$, are not equally easy to score. The " $p$ " contractions are the most consistent and unambiguous to score. The " $\mathrm{a}$ " contractions are the most difficult to score and can be confused with foraging movements of the head (Thomas, 1990). Expulsion of the gut contents is very easy to see, but sometimes results from passive release of pressurized gut contents and is not accomplished by visible active contractions of the " $x$ " muscles. In most cases, animals were observed at the highest magnification, $500 \times$, on the dissecting scope and every effort was made to be consistent from animal to animal. A simple computer program of our own design was used to record, while observing through a dissecting microscope, the exact time of occurrence of each step of the motor program for continuous periods of up to $90 \mathrm{~min}$. Activity records were made by pressing the appropriate key for each part of the motor program or other behavioral patterns when they occurred. Records were stored on computer disk for later analysis. These methods facilitated gathering large behavioral data sets with accuracy of better than $0.1 \mathrm{sec}$.

Simultaneous assays of defecation and feeding rates. C. elegans feed by pumping bacteria into the digestive tract via a muscular pharynx. The rate of pharyngeal pumping is a measure of food intake (Avery and Horvitz, 1990). Pumping rates were counted directly and recorded on the computer at the same time that defecations were recorded (see above). In most cases pumping rate was sampled for a test interval between defecations. These intervals were then averaged for a given animal. In other cases, pumping was counted continuously for the duration of the experiment (usually about $10 \mathrm{~min}$ ). Pumping rates sampled in these two ways did not differ significantly.

Assays on varying food concentrations. We determined that a liquid culture of OP50 with an absorbence of 0.6 at $\mathrm{OD}_{600}$ would dry into solid agar in $30 \mathrm{~min}$ and be the same density as a mature lawn (about $24 \mathrm{hr}$ old) seeded with normal dilute OP50 stock (Brenner, 1974). We made a $1 \times$ standard food stock by growing OP50 in rich LB broth, then centrifuging the bacteria and resuspending the pellet in an appropriate volume of distilled water to achieve the $\mathrm{OD}_{600}$ absorbence of 0.6 . The $1 \times$ standard was then diluted with distilled water as desired. Experimental plates were flooded with the diluted (or concentrated; in one experiment a $2 \times$ concentration was made) solutions of OP50 and excess fluid was immediately removed by inversion. Seeded plates were allowed to dry for $30 \mathrm{~min}$ before use in assays. These procedures ensured uniform and reproducible distribution of food on the plates. Worms were moved from lab stock plates with thick food lawns to the experimental plates with measured lawns and observations were initiated 


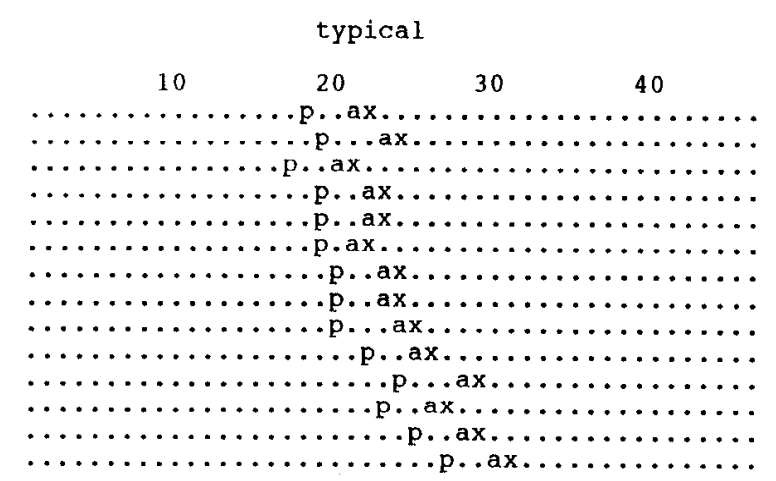

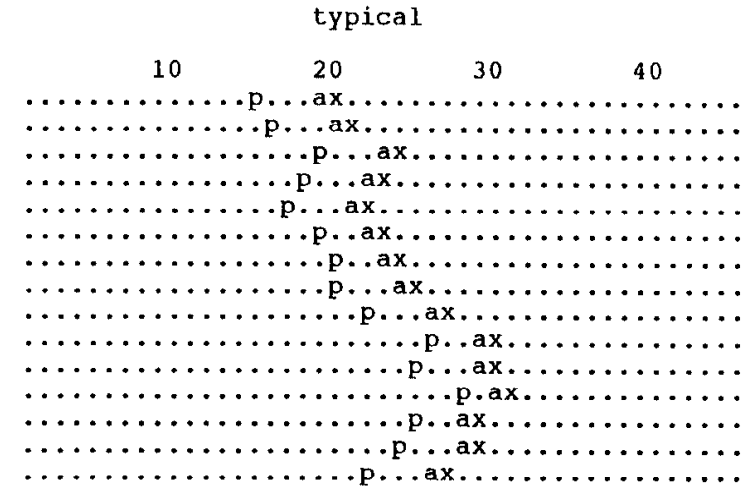

10

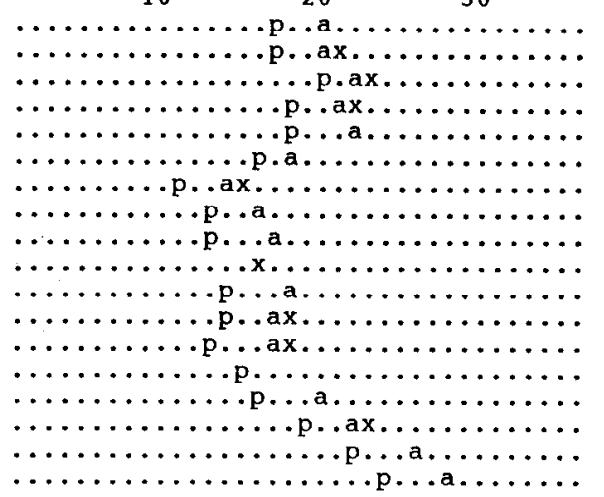

long

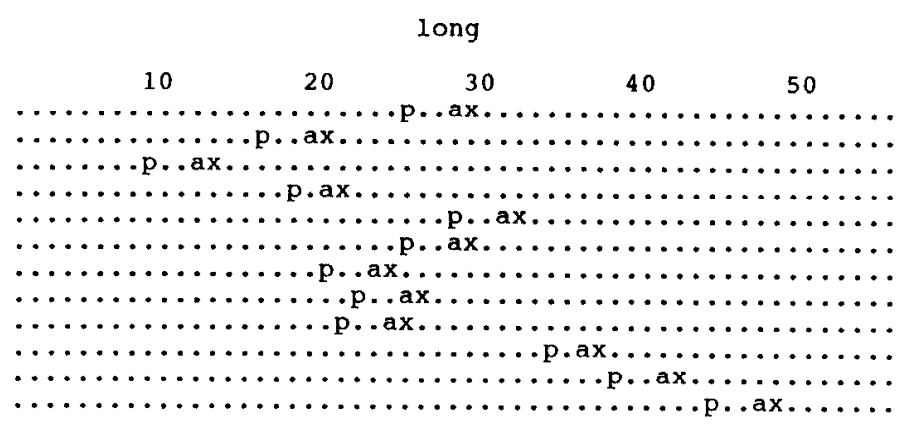

Figure 2. Individual animals have a stereotyped defecation rhythm: activity records from over 10 min of continuous observation on four different animals (animals 1, 13, 16, and 27 from the data set in Fig. 1, left), demonstrating the stereotyped motor program and its highly periodic activation. Top left, A typical young adult animal with an average defecation period of $46.7 \mathrm{sec}$ for 14 cycles, with an SD of $1.6 \mathrm{sec}$. All parts of the motor program were activated each cycle. Bottom left. An animal with a significantly shorter period than typical, 36.3 sec for 17 cycles, with an SD of $2.2 \mathrm{sec}$. In some cycles, parts of the motor program were not activated. Top right, Another typical record with a defecation period of $44.3 \mathrm{sec}$ for 14 cycles, and an SD of $2.4 \mathrm{sec}$. Bottom right, An animal with a longer than typical defecation period of $56.7 \mathrm{sec}$ for $12 \mathrm{cycles,}$ and a large SD of $7.2 \mathrm{sec}$. In all four panels parts of the motor program are abbreviated $p$, posterior body muscle contraction; $a$, anterior body muscle contraction; $x$, expulsion muscle contraction. Each dot represents one second of elapsed time. The width of each record has been adjusted to match the average period length.

after 5 or more minutes. Plates were discarded after another $60 \mathrm{~min}$ to minimize thickening of the lawn as the bacteria reproduced.

Temperature compensation experiments. Defecation rhythms of individual worms were assayed at various constant temperatures. Plates were placed in a circulating bath chilled by a Peltier device. Observations at temperatures higher than room temperature were made on plates placed in a small, well-insulated warm water bath. The temperature of the plate containing the worms was directly monitored using a small thermoprobe that was placed directly into the gelled agar. Data from experiments in which temperature fluctuated more than $1^{\circ} \mathrm{C}$ were not included in the analysis. Typically plates took less than $5 \mathrm{~min}$ to equilibrate with the bath temperature. Observations were made on worms after $20 \mathrm{~min}$ or more at the experimental temperature.

Food-leaving experiments. Worms were placed on plates with normal, thick bacterial lawns that were small in area. The smaller lawn area in proportion to the plate size meant that, in the course of foraging, worms left the bacterial lawn more frequently. Some food-leaving events were also observed in the course of observations on standard-sized lawns. The data recorded under the two conditions did not differ and they are combined in the results. The computer was used to record the exact time that the worms left and returned to food, in addition to the usual record of defecations and feeding rates.

Mechanosensory stimulation. Touch reset of defecation rhythms was measured after the fashion reported in Thomas (1990). Worms were lightly touched, just behind the pharynx, using an eyelash. In response, worms backed up but usually did not interrupt feeding. $\Lambda$ fter backing several body lengths they resumed normal foraging movements.

\section{Results}

Defecation is highly periodic

As previously reported (Croll and Smith, 1978; Thomas, 1990), we found that activation of the DMP was highly periodic. The cycle period variability within a single animal as well as between animals was small. To provide an accurate picture of the wildtype behavior and to provide a firm foundation for future work, we have collected a larger and more precise set of data on the defecation periodicity of wild-type C. elegans.

The mean defecation period for 27 different young-adult worms (11-17 cycles per animal) in the presence of plentiful food is shown in Figure 1 (left). The overall mean defecation period for these animals was $45.3 \mathrm{sec}$ (range, 36.3-56.8 sec), and the SD among the individual means was $4.3 \mathrm{sec}$. The SDs of the animals with the longer cycle periods (animals 21, 24, 26, and 27) tended to be longer than those of animals with cycle periods closer to or shorter than the average. The distribution of the defecation intcrvals for all 368 cycles recorded from these 27 animals is a nearly normal distribution centered about the mode $(45.1 \mathrm{sec})$ and the mean $(45.3 \mathrm{sec})$ for the population (Fig. 1, right).

Continuous defecation activity records for four animals (an- 
Figure 3. Variations in feeding rates do not correlate with variability in defecation period. Top, Average defecation periods plotted against average feeding rates (pharyngeal pumping rates) for 10 animals. Each animal was assayed for at least $10 \mathrm{~min}$ and pumping rate was sampled in the interval between each defecation. The scattered distribution of the data shows that increased feeding rates did not correlate with shorter defecation intervals. Broken lines mark mean defecation period and mean pumping rate for all 10 animals. Bottom, Continuous record of defecation intervals (open squares) and feeding rates (solid diamonds) in an individual animal. Fluctuations in either rate do not necessarily correlate (double arrow). The average defecation period for this animal was $46.2 \mathrm{sec}$, with an $\mathrm{SD}$ of $1.4 \mathrm{sec}$ over 13 cycles. The average pharyngeal pumping rate was 200 pumps/minute (corresponding to a pumping period of $0.3 \mathrm{sec} /$ pump) with an SD of 18.5
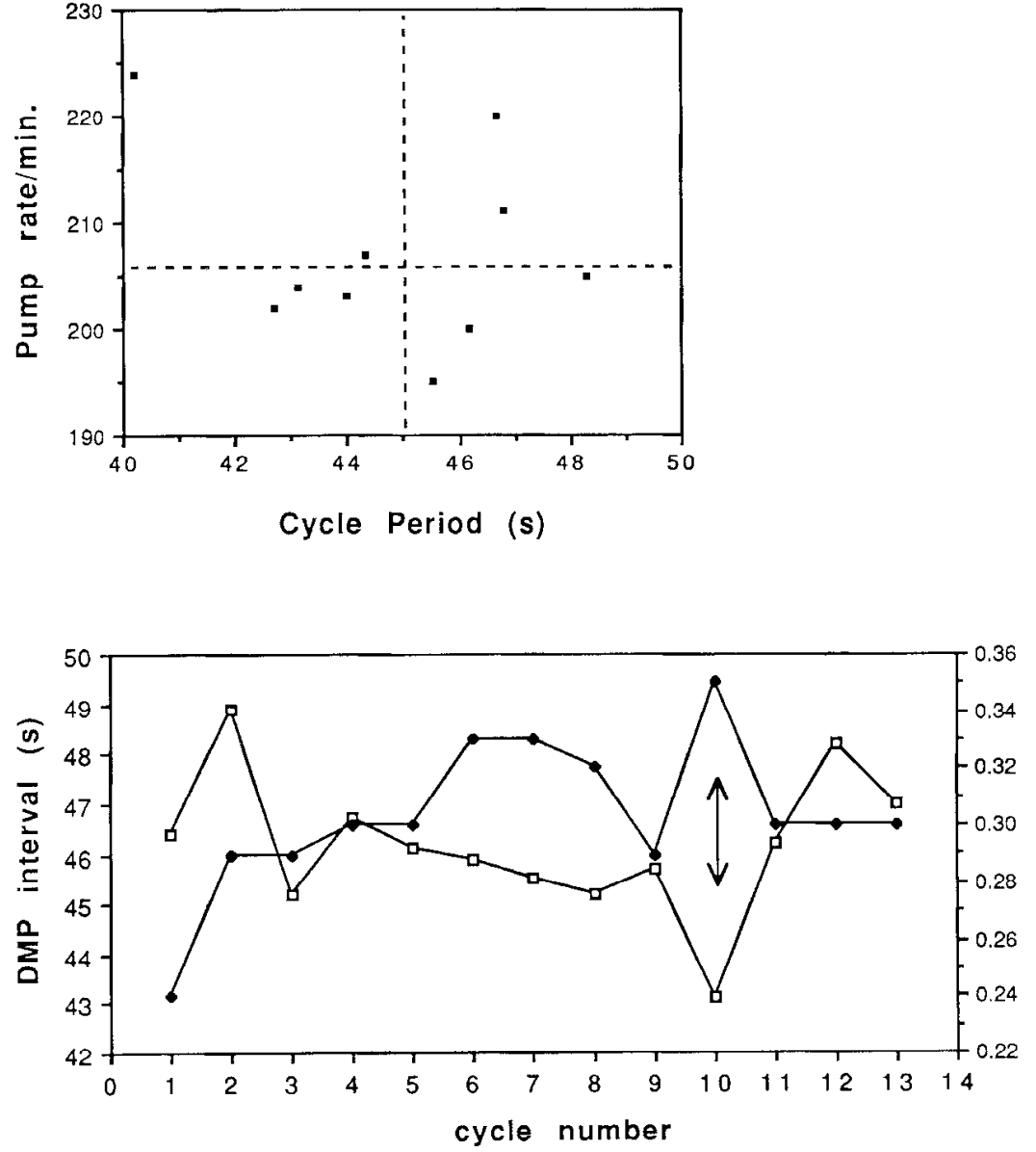

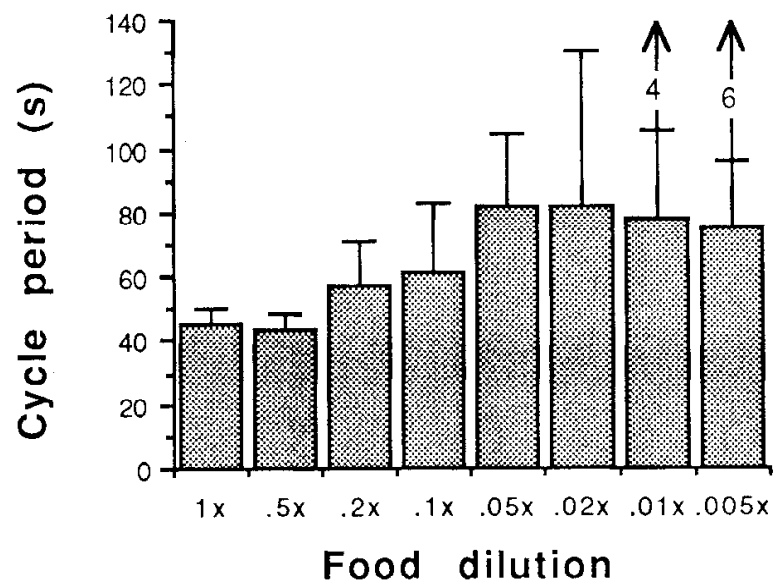

Figure 4. Food concentration affects defecation period: average defecation pcriod for 85 animals assayed on $2 \times$ to $0.005 \times$ dilutions of the concentration of food on which animals are typically raised $(1 \times$ defined as $A=0.6$ at $\mathrm{OD}_{600}$; see Materials and Mcthods). Average defecation period lengthened from about $45 \mathrm{sec}$ to over $80 \mathrm{sec}$ as food concentration was reduced 50 -fold $(0.02 \times)$. At further dilutions, average period was furthered lengthened, but a significant number of animals ( 4 of 13 for $0.01 \times ; 6$ of 13 for $0.005 \times$ ) stopped activating the motor program altogether (indicated by the arrows rising from the error bars), as animals do in the complete absence of food. imals $1,13,16$, and 27 from the 27 animal data set) are shown in Figure 2, where each step of the motor program is recorded as $\mathrm{p}, \mathrm{a}$, and $\mathrm{x}$, for posterior body-wall muscle contraction, anterior body-wall muscle contraction, and expulsion muscle contraction, respectively, and each dot is $1 \mathrm{sec}$ of elapsed time. The highly stereotyped structure of the motor program, $p$ followed by a few seconds delay then a and $x$, and the regularity of its activation are clearly demonstrated in the records. The upper panels show typical defecation activity records, with periods (left, $46.7 \mathrm{sec}$, SD of $1.6 \mathrm{sec}$; right, $44.3 \mathrm{sec}$, SD of $2.4 \mathrm{sec}$ ) close to the overall population average of $45.3 \mathrm{sec}$. The lower panels of Figure 2 show activity records for the shortest-period animal (left, $36.3 \mathrm{sec}$, SD of $2.2 \mathrm{sec}$ ) and longest-period animal (right, $56.8 \mathrm{sec}, \mathrm{SD}$ of $7.2 \mathrm{sec}$ ) of the 27 animal data set.

\section{Relation between feeding and periodicity}

Typically, behavioral observations of $C$. elegans are made in the presence of the $E$. coll they eat (see Materials and Methods). The $E$. coli are spread on solid nutrient agar and allowed to grow for about $24 \mathrm{hr}$. Under typical laboratory conditions the bacterial lawn is so thick that worms are immersed in their food and feeding continuously. C. clegans feed by pumping bacteria into their gut via a muscular pharynx that contains a grinding organ (Doncaster, 1962). The pharynx pumps at a fairly constant rate of about 200/min (Croll and Smith, 1978; Horvitz et al., 1982; Avery and Horvitz, 1989). 


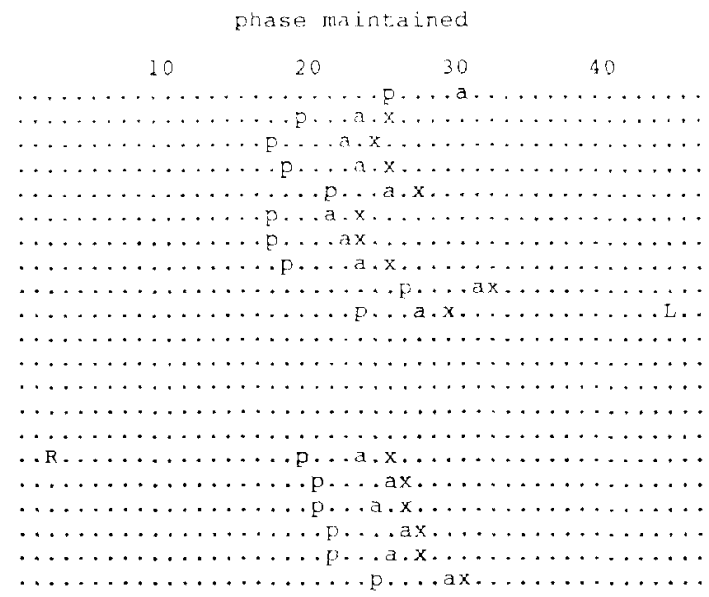

\section{phase shift}

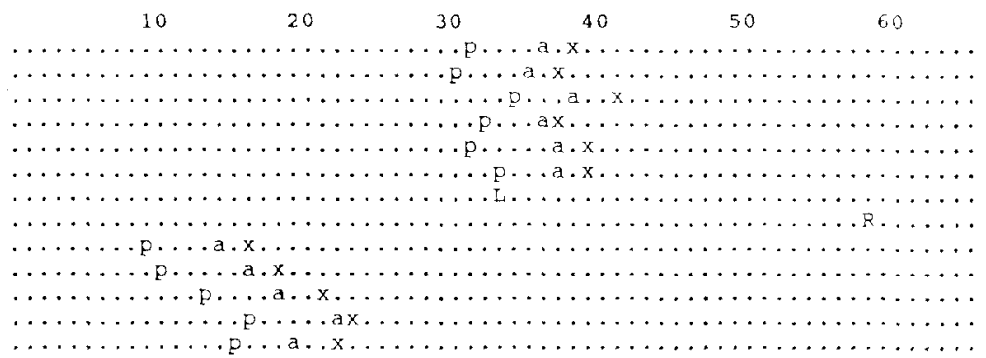

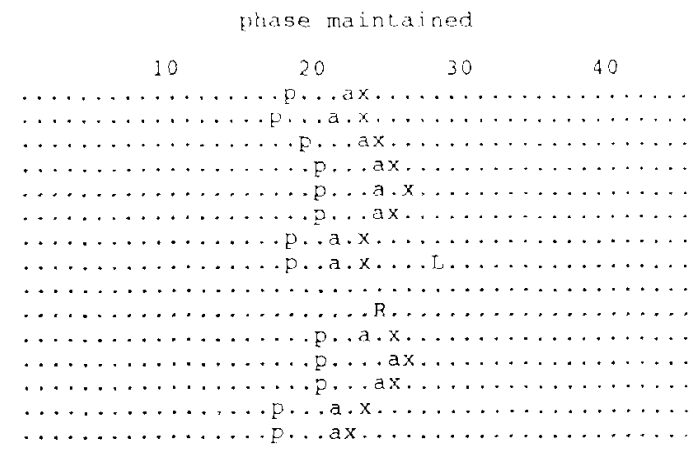

period change

$10 \quad 20 \quad 30$

$\ldots \ldots \ldots \ldots \ldots \ldots \ldots \ldots \ldots \ldots \ldots \ldots \ldots \ldots \ldots \ldots$ $\ldots \ldots \ldots \ldots \ldots \ldots \ldots \ldots \ldots \ldots \ldots \ldots$. $\ldots \ldots \ldots \ldots \ldots \ldots$

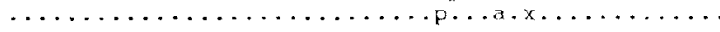
$\ldots \ldots \ldots \ldots \ldots \ldots \ldots \ldots \ldots \ldots \ldots \ldots$ $\ldots \ldots \ldots \ldots \ldots \ldots \ldots \ldots \ldots \ldots \ldots \ldots$ $\ldots \ldots \ldots \ldots \ldots \ldots \ldots \ldots \ldots \ldots \ldots, \ldots, \ldots_{\ldots} \ldots \ldots, \ldots \ldots$

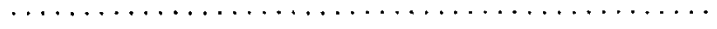
… $\ldots \ldots \ldots \ldots \ldots \ldots \ldots \ldots \ldots \ldots \ldots \ldots \ldots$

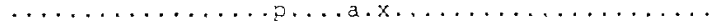

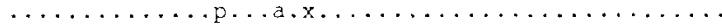

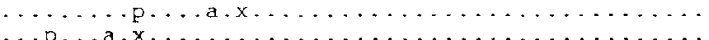
$\ldots$ p.

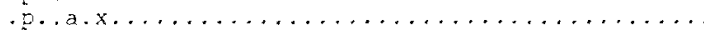

Figure 5. Animals maintain defecation rhythms in the absence of food and activation of the motor program: records from individual animals that left food for varying amounts of time. Top lefi. An animal that maintained phase after missing the equivalent of five defecation cycles. Top right, An animal that resumed defecating in phase after missing one activation. Bottom left, An example of the minority result in which the phase of the defecation rhythm was reset upon returning to food after missing the equivalent of two activations. Bottom right, An unusual animal that maintained phase upon retuning to food after missing the equivalent of two defecations, but changed period length upon resuming defecations. $L$, time animal left food; $R$, time that animal returned to food

Effect of feeding rates on defecation periodicity. The highly regular rate of feeding suggested that it could control, directly or indirectly, defecation periodicity. To look at the relationship between feeding and defecation, we measured pharyngeal pumping rates and defecation periodicity simultancously in individual animals. Each animal was assayed continuously for at least 10 min and mean pumping rates (calculated from timing 50 pumps in the interval between DMP activations) and mean defecation periods were calculated. Pumping rates and defocation periods were highly regular for the duration of each experiment, as were mean pumping rates and defecation periods between individual experiments (Fig. 3). The mean defecation periods varied over a range of about $20 \%$ between individual animals, while pumping rates varied only about $12 \%$. Variations in pumping rates did not correlate with variations in average defecation period, as shown by the scattered distribution in the upper panel of Figure 3. The lack of correlation between variations in pumping rate and defecation period was equally clear when the relationship was examined over time for an individual worm, as shown in the bottom panel of Figure 3. Sequential sampling of pumping rates (calculated from timing 50 pumps) in individual animals showed fluctuations in pumping rate that bore no discernible relation to variations in defecation period. The lack of correlation is most clearly demonstrated by the parts of the records in which fluctuations in pumping negatively correlated with fluctuations in the defecation rhythm (double arrow).

Effect of food concentration on periodicity. Although pumping rate per se in the presence of abundant food appears not to modulate defecation rhythms, we considered that food concentration might. We measured the defecation periodicity of individual animals on varying concentrations of food. A $\mathrm{i} \times$ concentration of food was defined by measuring the optical density of a thick mature hacterial lawn on which the worms are normally raised. All dilutions were then made in reference to this $1 \times$ density standard (see Materials and Methods). Pumping rates on lowered concentrations of food were similar to those observed for animals assayed in the presence of abundant food, except at the lowest food concentrations at which pumping became slow and sporadic (data not shown). The concentration of food affected DMP periodicity only when reduced many-fold from normal, causing the cycle period to lengthen and become more variable (Fig. 4). The cycle period lengthened to an average of $65 \mathrm{sec}$ when food concentration was reduced 10 -fold. The average cycle period lengthened further, to just over $80 \mathrm{sec}$, when food concentration was reduced 50 -fold. At dilutions of more than 50 -fold, the bacterial lawn was barely visible and a significant number of worms began to behave as they do when food was absent; they foraged very rapidly and defecated very 


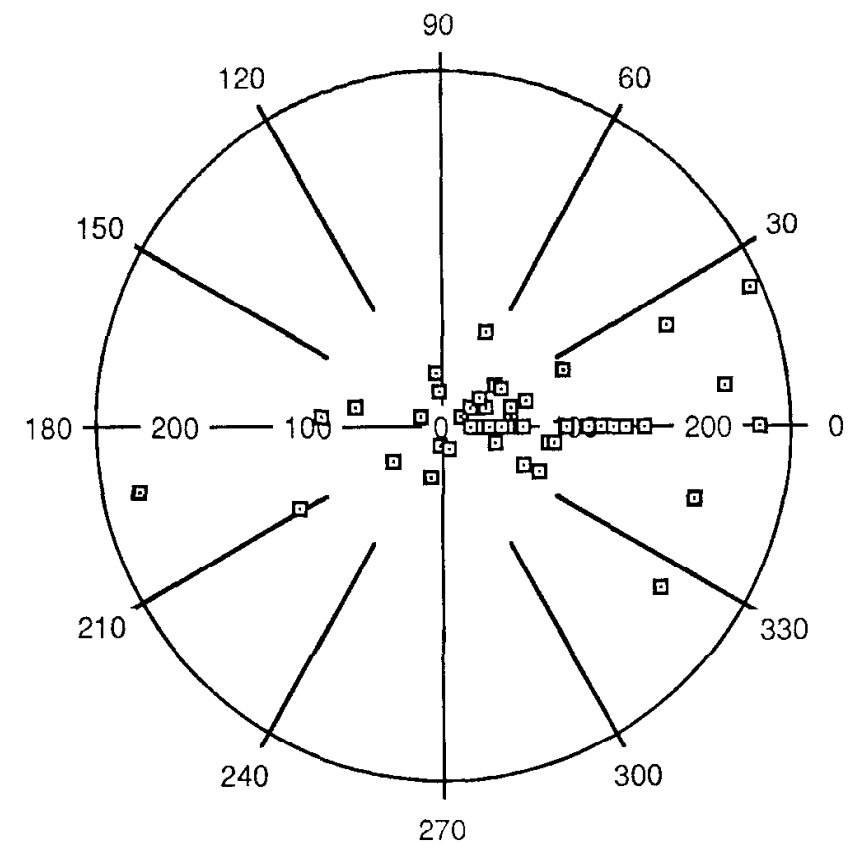

Figure 6. Most animals maintain defecation rhythms irrespective of the time spent away from food: phase relation data for 58 food-leaving events. The radial axis is the time each animal spent away from the food in seconds (up to $260 \mathrm{sec}$ ). The phase angles, from 0 to $360^{\circ}$ (representing the full $45 \mathrm{sec}$ cycle), show the phase shift recorded for each food-returning event when defecations resumed. In 14 cases (24\%) there was no phase shift at all. In $70 \%$ of the cases the phase shift was within 1 SD, and in $80 \%$ of cases within 2 SDs.

infrequently (less than once in $300 \mathrm{sec}$ ). These results indicate that food concentration, in contrast to feeding rate, does modulate the length of the DMP cycle period.

\section{Clock properties}

The highly periodic activation of the DMP, despite variations in feeding rates and food concentration, suggested that an endogenous clock, rather than an environmentally triggered reflex. might control DMP rhythms. To test this possibility we analyzed defecation rhythms under conditions that altered the expression of the DMP.

Maintenance of DMP rhtthms when feeding is interupted. In the presence of food, pumping and defecation rates are highly consistent, but these rhythms are interrupted when worms are away from food. In the course of collecting a large set of data on wild-type DMP cycles, we observed that worms spontaneously leave the bacterial lawn. When a worm left food it stopped pharyngeal pumping after several seconds, and while away from food did not activate the DMP. The cessation of pumping and DMP activations was also observed when worms were removed from food with a wire worm pick. However, the pick method of moving worms severely disrupts a variety of behavioral patterns, including locomotion, feeding, and defecation rhythms. Thus, the following observations were made on worms that had spontaneously left and returned to food.

Typically, a worm that left the food swam on the foodless agar surface for a few seconds to several minutes before returning to the bacterial lawn to resume feeding and defecating. The upper panels in Figure 5 show records from individual animals that left the food for different amounts of time and, upon returning to food, resumed defecating in phase with their previous

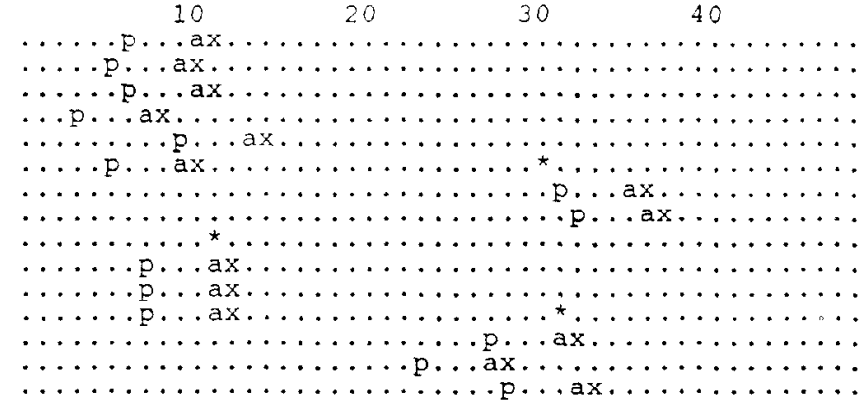

Figure 7. Stimulation of the light touch mechanosensory system resets defecation rhythms: an example of a wild-type animal's responses to three scquential light touch stimulations. At each touch $\left(^{*}\right)$ the animal responded by activating subsequent defecations in phase with delivery of the stimulation.

rhythm. The lower left panel of Figure 5 illustrates the minority result ( 11 of 58 cases) in which the phase of the rhythm clearly changed upon returning to the food, and the period slightly lengthened.

The polar plot (Fig. 6) shows time away from food versus the shift in phase upon returning to food for 58 different foodleaving events. The distribution of the phase shifts is clearly nonrandom and clusters near zero. In $70 \%$ of cases, individuals reactivated the DMP within 1 SD of the previously established rhythm, and in $80 \%$ of cases reactivation was in phase within 2 SDs. These results indicate that phase is usually maintained in the absence of food, when the pharynx is not rapidly pumping and the DMP is not being expressed.

DMP rhythm is reset by mechanosensory stimulation. Further evidence for an endogenous pattern generating mechanism was obtained from the results of phase-resetting experiments. We achieved phase resetting, as previously reported (Thomas, 1990), by activating the well-characterized neural circuit mediating response to light touch. When touched lightly with an eyelash, worms respond by moving away from the source of the stimulation. The principal sensory neurons, interneurons, and motor neurons mediating this response in $C$. elegans have been well characterized (Chalfie et al., 1985).

At various times in the defecation cycle we touched worms with an eyclash. Worms responded by backing but did not always interrupt feeding. In agreement with previous results (Thomas, 1990), light touch caused the DMP activation to reset phase to the lime of stimulation. Reset of the rhythm was achieved irrespective of the time of touch relative to DMP activations. An example of an animal undergoing a series of touch-reset stimulations is shown in Figure 7. The reset of the DMP rhythm to tince zero, irrespective of when the stimulation was applied, implies a strong input from the mechanosensory system to the putative defecation clock.

DMP rhythms are partially temperature compensated. Most biological processes are highly temperature dependent, typically doubling in rate with every $10^{\circ} \mathrm{C}$ temperature elevation within the physiological range. Circadian clocks have the property of being temperature compensated, even for plants and poikilothermic animals (Sweeney and Hastings, 1960). If circadian clocks were not temperature compensated the time-keeping function would fluctuate with daily and seasonal temperature changes. We tested whether the DMP clock function is temperature compensated by measuring defecation rhythms of worms feeding at various constant temperatures ranging from $13^{\circ} \mathrm{C}$ to $30^{\circ} \mathrm{C}$. The 


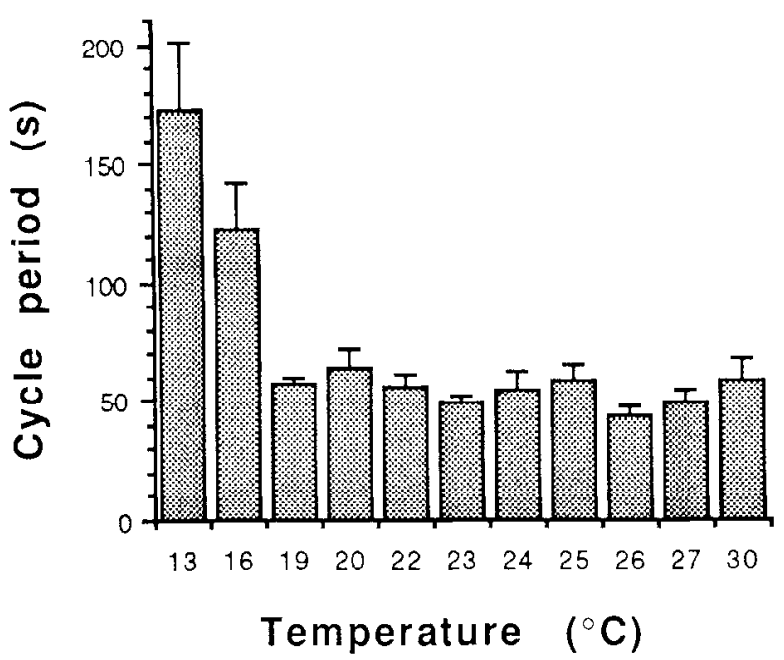

Figure 8 . The defecation clock is temperature compensated. Defecation rhythms were measured on 34 animals assayed at temperatures ranging from $13^{\circ} \mathrm{C}$ to $30^{\circ} \mathrm{C}$. Defecation rhythms were similar in the temperature range of $19-30^{\circ} \mathrm{C}$, varying only slightly from the baseline value of $45 \mathrm{sec}$ at $23^{\circ} \mathrm{C}$. Animals assayed at $16^{\circ} \mathrm{C}$ and $13^{\circ} \mathrm{C}$ had significantly longer defecation cycles, indicating a partial loss of temperature compensation. Error bars represent SD of the means of individual animals assayed for at least $10 \mathrm{~min}$ each.

rate of DMP activation was fairly well temperature compensated over the range of $19-30^{\circ} \mathrm{C}$ (Fig. 8). At $13^{\circ} \mathrm{C}$ and $16^{\circ} \mathrm{C}$ DMP activation period lengthened considerably, indicating a loss of temperature compensation in this low temperature range.

Cycle period is independent of the motor program. Several well-characterized fixed action patterns are controlled by pattern gencrators that consist entirely of interneurons (Thompson and Stent, 1976; Getting, 1983), while motor neurons appear to be integral members of the pattern generator circuit underlying other bchavioral patterns (Maynard and Selverston, 1975). Our evidence suggests that the defecation pattern-generating mechanism is independent of the activation of the motor program, since the phase was maintained when animals left the food and stopped defecating (Figs. 5, 6). In addition, most mutants defecive in the motor program did not have altered DMP periodicity. A few motor program mutants did have significantly faster periods than wild-type animals, but all the strains were still highly periodic (Thomas, 1990). An example of a mutant, called aex-?, that is defective in two of the three steps of the motor program, the anterior body-wall muscle contraction and the expulsion muscle contraction, is shown in Figure 9 (left). Note that the remaining posterior body muscle contractions, as well as the occasional activations of the defective parts of the DMP, occur with the same periodicity as wild-type animals.

The effect of killing motor neurons on DMP period. A role for the motor neurons AVL and DVB in activating the expulsion muscles (intestinal muscles, anal depressor, and anal sphincter) and the anterior body-wall muscles has been established by the analysis of mutants known to affect these neurons (McIntire et al., 1993a) and by killing these neurons with a laser microbeam (McIntire et al, 1993b). To test whether the AVL and DVB motor neurons are part of the pattern-generating circuit for defccation periodicity, we killed both cells in individual animals. In agreement with McIntire et al. (1993b), we found that killing the DVB and AVL motor neurons drastically reduced the number of anterior body-wall muscle contractions, and completely eliminated expulsion muscle contractions. The phenotype of the operated animals (Fig. 9, right) was very similar to the phenotype of the aex-2 mutant animals (Fig. 9, left). The operated animals missed most anterior body-wall muscle contractions (22\% of 249 DMP activations had an anterior body-wall muscle contraction) and all expulsion muscles contractions (0 expulsion muscle contractions observed for 249 DMP activations). The operated animals became very constipated because the gut contents were eliminated only occasionally, apparently when internal pressure caused a passive release (two examples, labeled "r" in Fig. 9). Despite the perturbation of the motor program and the severe bloating of the gut, operated animals had normal DMP activation rhythms (average period $=46.7 \mathrm{sec}, \mathrm{SD}=3.5$ $\mathrm{sec}$, for 242 cycles in 13 animals). These results show that the AVI and DVB motor neurons do not play an essential role in the rhythm-generating circuit. The results further suggest that
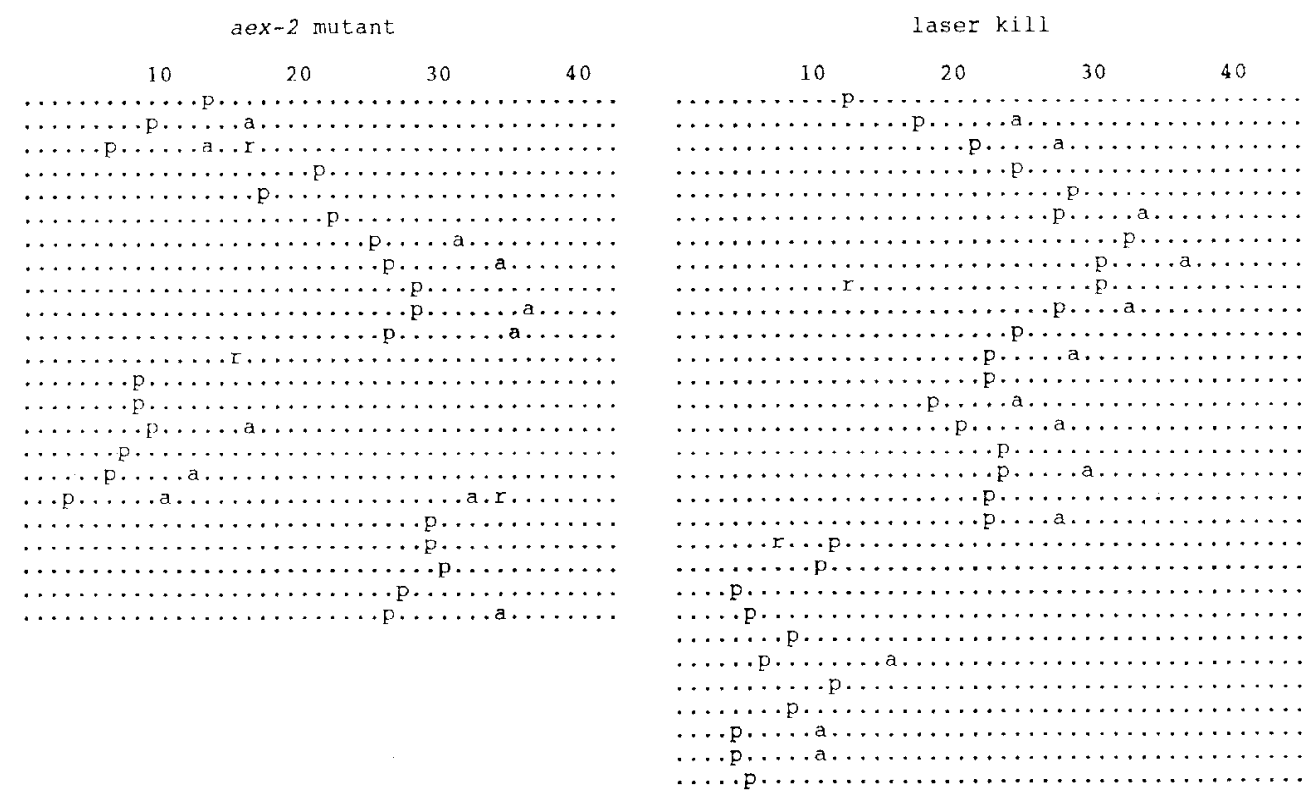

Figure 9. The timing of activations of defecations is independent of the normal expression of the motor program. Lefi, A defecation activity record from an aex- 2 mutant animal. Despite missing most $(55 \%)$ anterior body muscle contractions $(a)$ and all expulsions $(x)$, and becoming very constipated. this mutant still activates defecations, as represented by intact posterior body muscle contractions $(p)$, in a highly periodic manner. Average cycle period $43.7 \mathrm{sec}, \mathrm{SD}$ of $8.2 \mathrm{sec}$, for 22 cycles. The larger than typical SD is due to the reset of the rhythm in response to the explosive passive release of gut contents $(r)$. Right, Activity record from an adult animal in which the AVL and DVB motor neurons were both killed during the first larval stage. This animal missed all expulsions $(x)$ and $54 \%$ of anterior body-wall muscle contractions (a). The animal was constipated but had a defecation period of $41.3 \mathrm{sec}$ with an SD of $3.7 \mathrm{sec}$ for 28 cycles. The second passive release $(r)$ was violent enough to cause the reset of rhythm. 


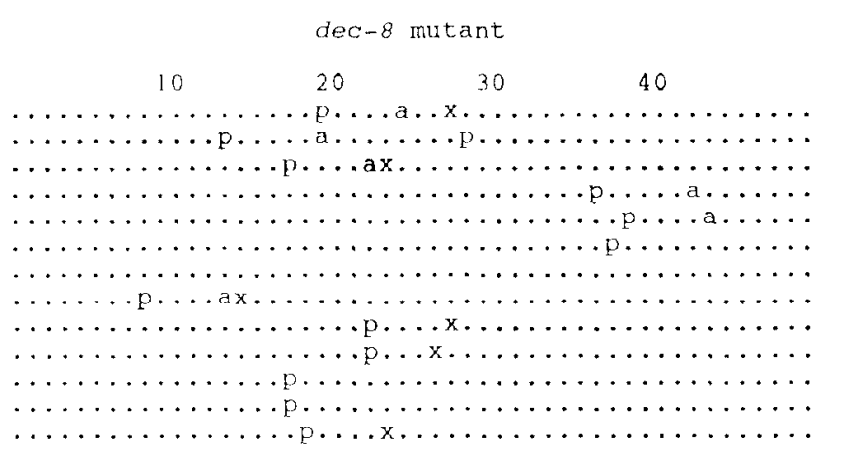

wild type

10

20

30

40

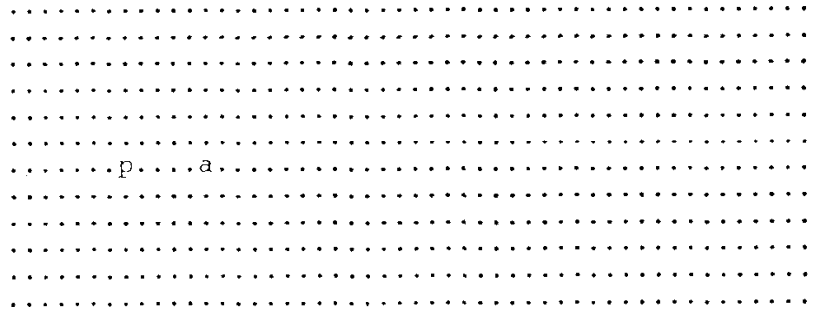

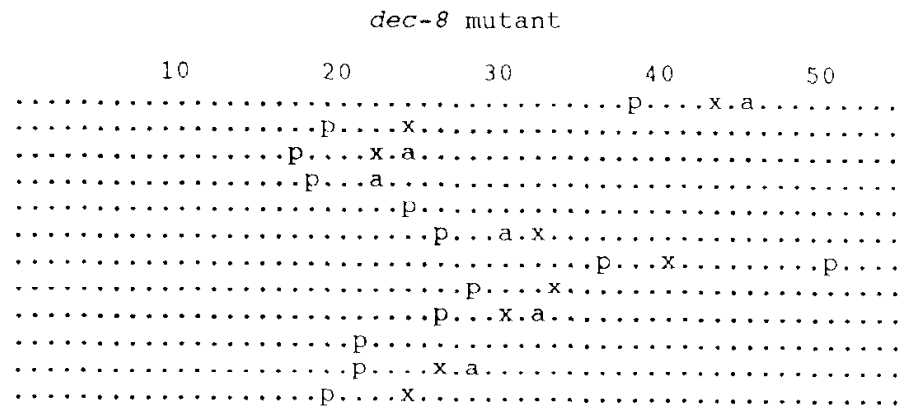

wild type

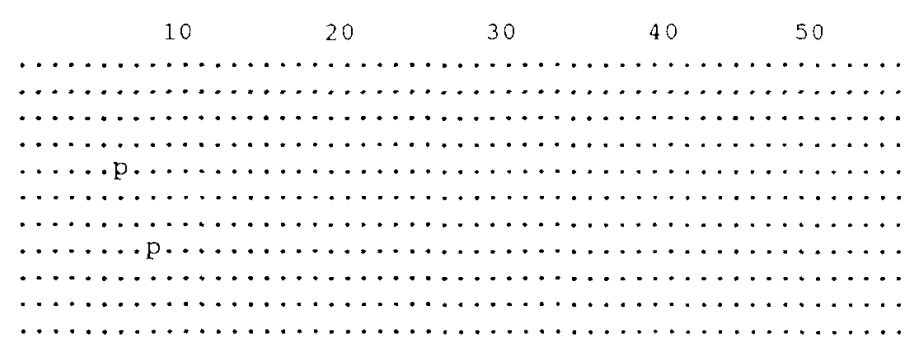

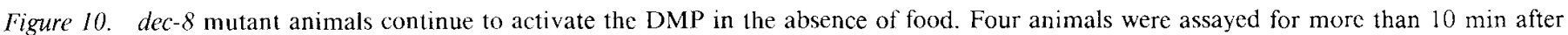

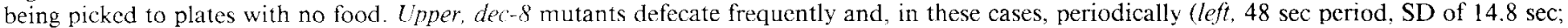
right, $46.7 \mathrm{sec}$ period, SD of $14.1 \mathrm{sec}$. Lower. Wild-type animals defecate very infrequently in the absence of food.

neither activation of, nor afferent feedback from, the anterior body-wall muscles and the expulsion muscles is required to generate normal periodicity.

Taken together, the motor program mutants and the AVL/ DVB kills indicate that activation of the muscles, activation of motor neurons, completion of the motor program, and release of gut pressure are not necessary for generation of the normal DMP rhythm.

\section{A mutation affecting the food modulation of cycle periodicity}

Although food modulates cycle periodicity our results also indicate that an internal clock functions independently of sensory input. Therefore, we were interested in studying mutations that affect food modulation. We reasoned, for example, that mutant animals that continued to activate the DMP in the absence of food might reveal intrinsic properties of the pattern generator such as its autonomous rate or timing accuracy. Additionally, a mutant in which the DMP was activated in the absence of food would support the existence of an endogenous clock.

We have identified a recessive mutation that causes a foodsensitive cycle period phenotype. We have named the gene dec- 8 (for defecation cycle) and the single mutant allele is designated sa200. The general responses of $d e c-8$ mutants to the bacterial lawn were normal: the animals remained on the lawn, swam and fed within it, and occasionally left food for briel periods, like the wild type. dec- 8 mutants fed normally, the pharynx pumped bacteria at a normal rapid rate (observed in 28 animals), and the animals grew at a normal rate. $d e c-8$ mutants exhibited slightly jerky or "nervous" locomotion, but this did not affect their ability to forage or mate effectively.

We compared the bchavior of dec-8 mutants and wild type in the absence of food. Wild-type worms, when removed from food, initially stopped pumping and defecating. After more than
10 min away from food, wild-type animals began sporadic pharyngeal pumping (observed in 19 animals removed from food). Defecation occurred very infrequently (27 DMP activations in $218 \mathrm{~min}$ of observation on 22 animals) and not rhythmically. After more than $2 \mathrm{hr}$ away from food, wild-type animals became constipated, presumably due to pharyngeal pumping drawing in liquid that bloated the gut in the absence of frequent defecations (Liu and Thomas, unpublished observations).

When first removed from food, $d e c-8(s a 200)$ animals, like the wild type, ceased feeding and activating the DMP. In marked contrast to wild type, dec-8(sa200) animals resumed cycling (20 of 23 animals), after about $20 \mathrm{~min}$ away from food, with an average DMP activation period of $91.3 \mathrm{sec}(\mathrm{SD}-36.9 \mathrm{sec}, 158$ cycles), and after more than $2 \mathrm{hr}$ away from food they were still not constipated. Some dec- 8 mutants activated the DMP highly periodically in the absence of food (Fig. 10, upper panels). These results suggest that the mechanism preventing defecation in the absence of food is not functioning normally in dec-8/sa200) animals.

In addition to activating the DMP constitutively, dec-8(sa200) animals had an unusual phenotype in the presence of abundant food; the DMP was activated nearly twice as often as in wildtype animals, and the structure of the periodicity was very different (Fig. 11). The principal dec-8 cycle, characterized by a normally timed and ordered DMP activation with strong muscle contractions, was followed by a second activation of the DMP ( $76 \%$ of 273 cycles in 28 animals). This "echo" activation often lacked the anterior body-wall muscle contraction or the expulsion muscle contraction part of the motor program, or the two occurred in reverse order, and all DMP muscle contractions were usually weaker than those of the principal cycle. The echo occurred from 10 to $17 \mathrm{sec}$ (average latency, $13.1 \mathrm{sec}$ for 207 echoes) after the principal DMP activation finished. 


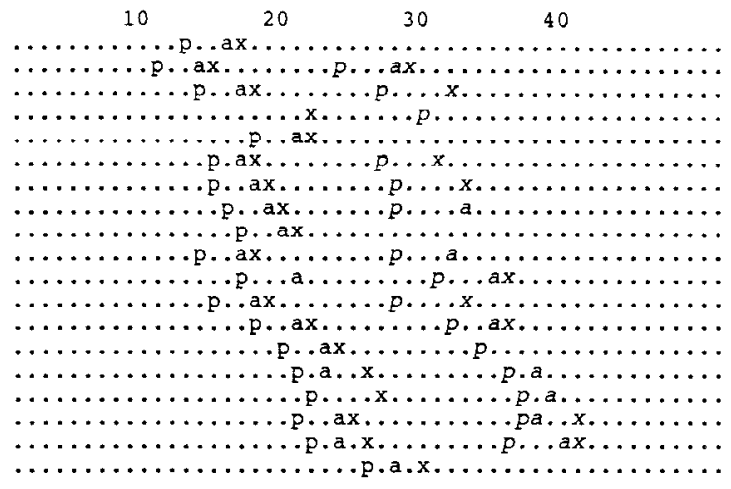

Figure 11. dec-8 mutant animals exhibit tandem activations of the motor program in the presence of ample food: defecation activity record from a young adult dec-8(sa200) animal on food. The principal activation (roman type) of the motor program is followed by an echo activation ( 15 of 19 activations). The echo activations (italic type) were weaker and the motor program was more variable.

The tandem activations of the DMP might be due to the activity of two independent pattern generators, as has been suggested for "split" activity in circadian rhythms (Earnest and Turek, 1982), or to dual activation of the DMP by the activity of a single pattern generator. If the principal DMP activation and the echo activation were produced by independent oscillators, each oscillator might have a different sensitivity to food and the interval between the principal and the echo DMP activation might depend on food concentration. As shown for wild-type animals (see Fig. 4), dec-8(sa200) animals' principal cycle period lengthened with decreasing concentration of food (Table 1). The latent period between principal DMP activation and echo activation remained fairly constant, in the 10-17 sec range. However, the frequency of echo activations decreased significantly with decreased concentrations of food. Thus, the presence of the echo activation appears to depend both on the presence of food and activation of the principal DMP, suggesting that it is not under the control of an independent pattern generator.

\section{Discussion}

Evidence that an endogenous clock controls DMP activations Throughout the animal kingdom fixed action patterns of the sort underlying $C$. elegans defecation have been shown to be controlled by neuronal pattern generators, rather than by simple reflex arcs (Delcomyn, 1980). Several lines of evidence support our hypothesis that an endogenous clock controls defecation periodicity. The most convincing evidence is our finding that animals that stop cxpressing the bchavior in the absence of food resume defecating in phase with their previous rhythm upon returning to food. The maintenance of the rhythm indicates that the putative clock continues to run in the absence of expression of the behavior and when away from the sensory stimulation of food. Sustaining a physiological rhythm in the absence of modulatory or entraining stimuli is a characteristic of other biological clocks. For example, circadian rhythms persist in the absence of environmental rhythms of light and other stimuli (Bunning, 1973).

We have shown that the putative defecation clock can be reset by gentle touch stimulation, mediated by a well defined mechanosensory neural circuit (Chalfie et al., 1985). Light touch stim-

\begin{tabular}{|c|c|c|c|c|}
\hline $\begin{array}{l}\text { Food } \\
\text { dilution }\end{array}$ & $\begin{array}{l}\text { Principal } \\
\text { period (sec) }\end{array}$ & $\begin{array}{l}\text { Echo } \\
\text { present }\end{array}$ & $\begin{array}{l}\text { Interval } \\
\text { to echo } \\
\text { (sec) }\end{array}$ & Animals \\
\hline $1.0 \times$ & 52.0 & $76 \%$ & 13.1 & $n=28$ \\
\hline $0.1 \times$ & 56.8 & $95 \%$ & 13.1 & $n=4$ \\
\hline $0.01 \times$ & 62.8 & $77 \%$ & 13.3 & $n=7$ \\
\hline $0.005 \times$ & 122.9 & $40 \%$ & 13.5 & $n=9$ \\
\hline $0.001 \times$ & 117.5 & $33 \%$ & 15.5 & $n=19$ \\
\hline No food & 147.6 & $10 \%$ & 17.3 & $n=19$ \\
\hline
\end{tabular}

The data show that food concentration affects the frequency of echo activation but not their latency.

ulation, regardless of when delivered, had the effect of setting the clock back to zero time, causing subsequent defecations to occur in phase with the time of the touch (Fig. 6; Thomas, 1990). The reset of the defecation clock to zero contrasts with most circadian clocks that also shift phase (in response to light pulses), but where the magnitude of the shift depends on when the stimulus is delivered and seldom produces a complete reset (Aschoff, 1965). Shorter period "clocks" also have measurable phase properties. Several neuronal pattern generators controlling rhythmic behavioral patterns have been well studied (Selverston, 1985). The rhythms produced by these pattern generators can be reset by stimulating members of the pattern-gencrating neuronal circuit or upstream command neurons, but not by stimulating follower cells or neurons outside of the circuit altogether (Wilson, 1961; Getting et al., 1980). Our touch-reset results imply that the mechanosensory system has a very strong input to the putative defecation clock. We speculate that this input functions to inhibit the activation of the DMP when an animal is startled and needs to focus on locomotion.

\section{Alternative models}

The main objection to DMP rhythms arising from an endogenous source is the very strong modulation by food. We found that lowering food concentration lengthened the period between DMP activations, and that in the absence of food the DMP was very rarely activated. However, the food-leaving experiments discussed above indicate that feeding is not required for maintenance of the phase of the rhythm. In addition, careful simultaneous measurement of defecation periods and feeding rates revealed a lack of a strict correlation, and several striking instances of negative covariance. Moreover, the degree of correlation was variable within individual experiments and from animal to animal. 'T hese results make it difticult to understand how feeding rates alone could account for the very tight periodicity of defecation rhythms.

Cyclical gut distention has been proposed as a reflex trigger for defecation in other nematodes (Crofton, 1966). As previously pointed out (Thomas, 1990), gut distention is an unlikely mechanism for producing defecation rhythms since mutations in many different genes cause constipation by disrupting the motor program, but do not affect the periodicity of DMP activation. Our laser kills of the AVL and DVB motor neurons, which cause severe constipation as well, also did not alter the periodicity of DMP activations. The fact that constipated animals, independently produced by genetic and neuronal lesion, retained normal defecation rhythms indicates that cyclical gut pressure is dispensable for the production of defecation rhythms. 
Finally, the fact that $d e c-8(s a 200)$ animals continue to activate the motor program in the absence of food further suggests the existence of an endogenous pattern generator. A reasonable interpretation of the dec- 8 mutant is that it affects a mechanism that normally prevents the activation of the DMP in the absence of fond, perhaps to conserve energy. The mutant might be revealing the activity of the pattern generator in the absence of modulation. If that is the case, food appears to sharpen, not generate, defecation periodicity.

\section{The clock is independent of the motor program}

Neuronal pattern generators may consist entirely of interneurons, or motor neurons may be integral members (Maynard and Selverston, 1975; Paul and Mulloney, 1985). Sensory feedback often modulates a behavioral rhythm but is not generally essential for its production (Wilson, 1961; Delcomyn, 1980). Several lines of cvidence suggest that the motor neurons or expression of the motor program is not an integral part of the pattern-generating mechanism for defecation rhythms. Killing the motor neurons $\triangle$ VL and DVB in the same animal eliminated the contraction of expulsion muscles, and greatly reduced the occurrence of anterior body contractions, but did not affect defecation periodicity. Clearly neither AVL and DVB nor activation of the muscles they excite is required for normal timing of DMP activation. This result is corroborated by mutants that affect the same or other steps of the motor program and also still periodically activate the motor program (Thomas, 1990). The result that animals maintain the phase of their defecation rhythms in the absence of DMP expression, as previously discussed, also supports the idea that the motor program and the cycle generator are independent.

\section{dec-8 mutant animals}

The dec-8(sa200) animals have an intriguing food-dependent defecation phenotype. An hypothesis that explains the $d e c-8$ mutant phenotype is that the wild-type dec-8 gene functions to inhibit defecations in the absence of food. The phenotype in the presence of food may support this hypothesis as well. The echo activations that follow the principal activation of the motor program could also be due to the failure of an inhibitory mechanism, thus resulting in extra DMP activations. The echo occurred only following a principal DMP activation, and with a consistent latency, indicating that the ccho depends on the principal activation rather than being produced by an independent pattern generator. Our finding that lowering food concentration does not change the phase relation between the principal and echo activations, but decreases the occurrence of echo activations, further supports that there is only one oscillator responsible for activating the DMP.

We conclude that the timing of defecation in C. elegans is regulated by an endogenous clock that functions independently of expression of the motor program. Our detailed behavioral analysis provides a firm basis for further studies directed at identifying the cellular identity of the neuronal pattern generator and for characterizing mutations that affect the generation of defecation rhythms to elucidate the molecular mechanisms underlying the function of cellular oscillators.

\section{References}

Aschoff J (1965) Response curves in circadian periodicity. In: Circadian clocks (Aschoff J, ed), pp 95-111. Amsterdam: North-Holland.
Austin C, Short R (1972) Hormones in reproduction. New York: Cambridge UP.

Avery L, Horvitz HR (1989) Pharyngeal pumping continues after laser killing of the pharyngeal nervous system of $C$. elegans. Neuron 3:473485

Avery L, Horvitz HR (1990) Effects of starvation and neuroactive drugs on feeding in Caenorhabditis elegans. J Exp Zool 253:263-270.

Binkley S (1990) The clockwork sparrow: time, clocks, and calendars in biological organisms. Englewood Cliffs, NJ: Prentice-Hall.

Brenner S (1974) The genetics of Caenorhabditis elegans. Genetics 77 $71-94$.

Bunning E (1973) The physiological clock, 3d ed. New York: Springer.

Chalfie M, Au M (1989) Genetic control of differentiation of the Caenorhabditis elegans touch receptor neurons. Science 243:1027-1033.

Chalfie M, Sulston JE, White JG, Southgate E, Thomson JN, Brenner S (1985) The neural circuit for touch sensitivity in Caenorhabditis elegans. J Neurosci 5:956-964.

Crofton HD (1966) Nematodes. London: Hutchinson University Library.

Croll NA, Smith JM (1978) Integrated behavior in the feeding phase of Caenorhabditis elegans (Nematoda). J Zool (Lond) 184:507-517.

Delcomyn F (1980) Neural basis of rhythmic behavior in animals. Science 210:492-498.

Doncaster CC (1962) Nematode feeding mechanisms. I. Observations on Rhabditis and Pelodera. Nematologica 8:313-320.

Earnest DJ, Turek FW (1982) Splitting of the circadian rhythm of activity in hamsters: effects of exposure to constant darkness and subsequent re-exposure to constant light. J Comp Physiol 145:405411.

Edmonds LN, Adams KJ (1981) Clocked cell cycle clocks. Science 211:1002-1013.

Getting PA (1983) Neural control of swimming in tritonia. In: Neural origin of rhythmic movements (Roberts A, Roberts BL, eds), pp 89128. Cambridge: Cambridge UP.

Getting PA, Lennard PR, Hume RI (1980) Central pattern generator mediating swimming in tritonia. I. Identification and synaptic interactions. J Neurophysiol 44:15 I-164.

Gray J (1939) Aspects of animal locomotion. Proc R Soc Lond [Biol] 128:28-62.

Horvitz HR, Chalfie M, Sulston, JE, Evans PD (1982) Serotonin and octopamine in the nematode Caenorhabditis elegans. Science 216 $1012-1014$

Hoyle G (1982) Muscles and their neural control, pp 370-382. New York: Wiley.

Lorenz $\mathrm{K}$, Tinbergen $\mathrm{N}$ (1938) Taxis und Instinkthandlung in der Eirollbewegung der Graugans. Z Tierpsychol 2:1-29.

Maeda H, Yamagata A, Nishikawa S, Yoshinaga K, Kobayashi S, Nishi K, Nishikawa S-I (1992) Requirement of c-kit for development of intestinal pacemaker system. Development 116:3699-3715.

Maynard DM, Selverston AI (1975) Organization of the stomatogastric ganglion of the spiny lobster. IV. The pyloric system. J Comp Physiol 100:161-182.

McIntire SL, Jorgensen E, Horvitz HR (1993a) Genes required for GABA function in Caenorhabditis elegans. Nature 364:337-341.

McIntire SL, Jorgensen E, Horvitz HR (1993b) The GABAergic nervous system of Caenorhabditis elegans. Nature 364:334-337.

Paul DH, Mulloney B (1985) Nonspiking local interneuron in the motor pattern generator for the crayfish swimmeret. J Neurophysiol $54: 28-39$

Selverston AI (1985) Model neural networks and behavior. New York: Plenum.

Smith DA, Saldana R (1992) Model of the $\mathrm{Ca}^{2+}$ oscillator for shuttle streaming in Physarum polycephalum. Biophys J 61:368-380.

Sweeney BM, Hastings J (1960) Effects of temperature upon diurnal rhythms. Cold Spring Harbor Symp Quant Biol 25:87-113.

Thomas JH (1990) Genetic analysis of defecation in Caenorhabditis elegans. Genetics 124:855-872.

Thompson WJ, Stent GS (1976) Neuronal control of the heartbeat in the medicinal leech. II. Intersegmental coordination of heart motor neuron activity by heart interneurons. J Comp Physiol 111:281-307.

von Holst E (1937) Vom Wesen der Ordnung im Zentralvervensystem. Naturwiss 25:625-647.

Wilson DM (1961) The central nervous control of flight in a locust. J Exp Biol 38:471-490. 